\title{
Determination and application of a reasonable drainage rate of coalbed methane in vertical wells in the single-phase flow stage
}

\author{
Sen Yang ${ }^{1}$, Xiaoming $\mathrm{Ni}^{2}$, Xuebin $\mathrm{Tan}^{2}, *$, Zheng $\mathrm{Zhao}^{2}$, Peng Chen ${ }^{3}$ \\ ${ }^{1}$ Drilling Branch of Shanxi Xishan Coal and Electricity Group, Taiyuan 030053, Shanxi, China \\ ${ }^{2}$ School of Energy Science and Engineering, Henan Polytechnic University, Jiaozuo 454000, Henan, China \\ ${ }^{3}$ Shanxi Lanyan coalbed methane Group Co., Ltd., jincheng 048204, Shanxi, China
}

\begin{abstract}
The determination of a reasonable drainage rate of coalbed methane (CBM) in vertical wells in the single-phase flow stage can provide maximise the transmission of water pressure over distance. Based on the principle of effective stress and Darcy's law, a mathematical model for dynamic changes of the permeability in the single-phase flow stage was established; on this basis, the relationship between permeability and threshold pressure gradient was experimentally attained; according to the linkage of changes of the transmission distance of water pressure, permeability, and pressure drop in the wellbore in the drainage process, a mathematical model for a reasonable reduction rate of the working fluid level in the single-phase flow stage taking the change of the permeability into account was established. The accuracy of the mathematical model was verified according to practical drainage data from CBM wells in Daning Block in Qinshui Basin, Shanxi Province, China. The results show that the rate of pressure drop decreases in a negative exponential manner with the increase of the drainage time. Different rates of pressure drop were required in coal reservoirs with different permeabilities; when keeping other conditions constant, the larger the permeability of coal reservoirs, the lower the threshold pressure gradient and the lower the rate of daily pressure drop. The research results provide a theoretical basis and reference for the reasonable drainage system in the single-phase flow stage.
\end{abstract}

\section{Introduction}

Ground vertical wells for coalbed methane (CBM) are designed to realise the desorption and production of CBM through water drainage and pressure drop, that is, methane in coal seams can be desorbed and produce gas only when water in coal seams flows and the pressure of coal reservoirs decreases to a certain extent. Whether water in coal seams flows or not matters the gas production. In the single-phase flow stage, the rate of change of the effective stress is controlled by controlling the drop rate of the working fluid level, thus influencing the transmission distance of water pressure. To explore the rate of reduction of fluid levels in the single-phase flow stage of lowpermeability coal seams, researchers have conducted numerous studies. Based on Darcy's law, some researchers established a mathematical model for the average drop rate of fluid levels in the single-phase flow stage according to practical drainage data ${ }^{[1-3]}$; however, the drop rate in the single-phase flow stage may vary, so the average drop rate of fluid levels fails to guide field production. Based on field production data, some researchers defined certain indices (such as the daily water production index, that is, the ratio of average drop rate of working liquid level to daily water production) to constrain the reasonable drop rate of the working fluid level during drainage from CBM wells ${ }^{[4-6]}$. Many scholars perform analysis from a statistical perspective. The analytical results are relatively adaptive to guiding operations in a certain area while providing limited guidance for other areas without CBM production data. Using some numerical simulation software (Comet 3.0 and Eclipse E300) for the production capacity, some researchers conducted parameter reversion for reservoirs through historical matching ${ }^{[7-9]}$. By doing so, the influences of different drainage rates on the production capacity of CBM wells and the optimal drainage system were attained. The rationality of the parameter selection affects the results.

To ascertain the rationality of the drop rate of the level of working fluid in the drainage process in the singlephase flow stage of CBM wells, a mathematical model for the reasonable drop rate was established by considering the linkage of changes of the transmission distance of water pressure, permeability and the level of working fluid during drainage from CBM wells. Based on practical production data, the rationality of the drop rate of fluids in the single-phase flow stage was evaluated, expecting to provide a theoretical basis for determining a reasonable drainage system in the single-phase flow stage of CBM wells.

\section{Research methods}

In its original state, coal matrix together with water, gas

* Corresponding author: Xuebin Tan, E-mail: c.b_txb@foxmail.com 
and pore/fracture system in coal seams jointly bears the overburden pressure. In the initial drainage stage of CBM wells, the stress on the coal matrix increases and pores/fractures in coal seams are narrowed, or even closed, when water in coal seams starts to flow towards the wellbore. As a result, the permeability of coal seams decreases and the resistance to flow increases; as drainage continues and the radius of influence grows, the pressure difference required for water flow to the furthest end increases, as evinced by a reduction in the level of working fluid. If the drop rate of the level of working fluid is too large, the permeability decreases too much and the resistance to flow rises rapidly. Consequently, the transmission distance of water pressure in the single-phase flow stage is affected and thereby the gas production of CBM wells. If the level of working fluid drops so slowly that cannot satisfy the minimum pressure difference required for water flow at the far end, the water therein will fail to flow and the actual transmission distance of water pressure is restricted. It is necessary to guarantee not only the continuity of flow in the drainage process but also maximise the transmission distance of water pressure in the single-phase flow stage. The drop rate of the level of working fluid satisfying these two conditions is the most reasonable drainage rate. The modelling of the reasonable drainage rate in the single-phase flow stage can be described as follows:

(1) Based on Darcy's law and the drainage characteristics of vertical CBM wells, a mathematical model for the permeability change within any range of influence during drainage in the single-phase flow stage is established according to the principle of effective stress.

(2) The threshold pressure gradients under different permeabilities are tested experimentally to reveal the relationship between the permeability and the threshold pressure gradient.

(3) According to the relationships in changes of the bottom-hole pressure, the transmission distance of water pressure and the permeability, the dynamic change of the permeability is successively substituted into equations for related parameters. In this way, a mathematical model for a reasonable drop rate of the fluid level in the single-phase flow stage at any time can be obtained.

\section{Establishment of the mathematical model for the reasonable drop rate in the single-phase flow stage}

\subsection{Mathematical model for the change in permeability in the single-phase flow stage}

In the initial drainage stage of vertical CBM wells, the reservoir pressure is higher than the critical desorption pressure. In this case, it is in the single-phase flow stage. It is supposed that coal seams appear as homogeneous, equal-thickness and infinite reservoirs; the recharge of water from surrounding rocks is ignored during the drainage. The initial height of the level of working fluid is hc and thus the reservoir pressure in the original state is calculated as follows:

$$
P_{c}=\frac{\rho g h_{c}}{10^{6}}
$$

With the outflow of water in the drainage process, the effective stress on coal reservoirs within the range of influence of the water pressure transmission increases. It is supposed that the level of working fluid of the wellbore decreases to height $\mathrm{hn}$ after drainage time tn. On this condition, the increment of the effective stress on coal reservoirs around the wellbore is expressed as follows:

$$
\Delta \sigma=\frac{\alpha \rho g\left(h_{c}-h_{n}\right)}{10^{6}}
$$

where, $\Delta \sigma$ and $\alpha$ refer to the increment (MPa) of the effective stress and the effective stress coefficient, respectively.

It is supposed that the water flow in coal seams conforms to Darcy's law with the threshold pressure gradient. According to the seepage principle[10-11], the pressure at a position $r$ from the wellbore within the range of influence of the water pressure in the drainage process of vertical CBM wells can be expressed as follows:

$$
p_{\mathrm{r}}=p_{c}-\frac{p_{c}-p_{w}}{\ln \frac{R_{e}}{r_{w}}} \ln \frac{R_{e}}{r}
$$

where, $p_{r}, p_{w}, \mathrm{Re}$, and $\mathrm{r}_{\mathrm{w}}$ refer to the pressure $(\mathrm{MPa})$ at a point $\mathrm{r}$ from the wellbore at a certain time in the drainage process, the bottom-hole pressure $(\mathrm{MPa})$ at the time, the radius $(\mathrm{m})$ of influence of the water pressure transmission at the time, and the outer radius (m) of the production casings.

According to Equation (3), the pressure drop at distance $r$ from the wellbore at that time in the drainage process can be calculated, that is,

$$
\Delta \mathrm{p}_{\mathrm{r}}=\frac{\mathrm{p}_{\mathrm{c}}-\mathrm{p}_{\mathrm{w}}}{\ln \frac{\mathrm{Re}}{r_{w}}} \ln \frac{\mathrm{Re}}{r}
$$

where, $\Delta \mathrm{p}_{\mathrm{r}}$ represents the pressure drop $(\mathrm{MPa})$ at distance $r$ away from the wellbore at that time during the drainage process;

According to the pressure transmission behaviour of low-permeability reservoirs[12-13], the relationship between the transmission distance of water pressure and the drainage time in the drainage process is obtained as:

$$
R_{e}=\sqrt{\frac{24 \mathrm{k}_{d w} \mathrm{vt}_{\mathrm{n}}}{6 \mathrm{v} \varphi \mu C_{\mathrm{t}}+4 \mathrm{k} C_{\mathrm{t}} \lambda}}
$$

where, $\mathrm{kdw}, t_{\mathrm{n}}$, and $\Phi$ denote the dynamically changing permeability $(\mathrm{mD})$ of coal reservoirs in the single-phase flow stage, the drainage time (d) in the single-phase flow stage, and the porosity (a decimal fraction) of coal reservoirs, respectively; $\mu, \mathrm{Ct}$, and $\lambda$ denote the viscosity $(\mathrm{mPa} \cdot \mathrm{s})$ of water, the total compressibility (MPa-1) of the formations, and the 
threshold pressure gradient $(\mathrm{MPa} / \mathrm{m})$, respectively.

Numerous studies have proved the following relationship between the effective stress and the permeability ${ }^{[14]}$ :

$$
k_{d w}=k_{0} \bullet e^{-3 C_{p} \Delta \sigma}
$$

where, $k_{0}$ and $C_{P}$ denote the original permeability $(\mathrm{mD})$ of coal reservoirs and the pore volume compressibility $\left(\mathrm{MPa}^{-1}\right)$, respectively.

By simultaneously calculating Equations (1), (2), (4), and (6), the permeability of coal reservoirs within the range of influence of the transmission distance of water pressure during drainage is expressed as follows:

$$
\mathrm{k}_{\mathrm{dw}}=\mathrm{k}_{0} \cdot\left(\frac{\mathrm{Re}}{r}\right)^{-3 C_{p} \alpha \frac{p_{c}-p_{w}}{\ln \frac{\mathrm{Re}}{r_{w}}}}
$$

where, assuming $-3 C_{p} \alpha \frac{p_{c}-p_{w}}{\ln \frac{\mathrm{Re}}{r_{w}}}=m$, the mean of

the dynamically changing permeabilities of reservoirs within the radius of influence of the water pressure transmission during drainage can be further expressed as follows:

$$
\overline{\mathrm{k}}_{\mathrm{dw}}=\frac{1}{\mathrm{Re}} \int_{r w}^{\mathrm{Re}} k_{0} \cdot\left(\frac{\mathrm{Re}}{r}\right)^{m} d r
$$

where, $\overline{\mathrm{K}}_{\mathrm{dw}}$ denotes the mean $(\mathrm{mD})$ of the changing permeabilities within the range of influence of the water pressure during drainage in the single-phase flow stage.

Integral can be obtained as follows:

$$
\overline{\mathrm{k}}_{\mathrm{dw}}=\frac{\mathrm{k}_{0} \cdot \mathrm{Re}-\mathrm{k}_{0} \cdot \operatorname{Re}{ }^{-3 C_{p} \alpha \frac{p_{c}-p_{w}}{\ln \frac{\mathrm{Re}}{r_{w}}}} \cdot r_{w}{ }^{3 C_{p} \alpha \frac{p_{c}-p_{w}}{\ln \frac{\mathrm{Re}}{r_{w}}+1}}}{\left.3 C_{p} \alpha \frac{p_{c}-p_{w}}{\ln \frac{\mathrm{Re}}{r_{w}}}+1\right)\left(\mathrm{Re}-r_{w}\right)}
$$

\subsection{Relationship between the permeability and the threshold pressure gradient}

Ferrarin (1951) proposed that flow cannot happen unless the pressure gradient exceeds a certain critical value (the threshold pressure gradient). There are many methods (including experimental test, numerical simulation, steady state well testing, and transient well testing) to determine the threshold pressure gradient. Here the threshold pressure gradient is tested by applying the pressure difference-flow method.

The method for calculating the threshold pressure gradient of sandstone proposed by Wu et al ${ }^{[15]}$. is adopted and the gas seepage equation without considering the threshold pressure gradient is

$$
v=\frac{k\left(p_{1}^{2}-p_{2}^{2}\right)}{2 p_{0} \mu L}
$$

where, $\mathrm{v}, \mathrm{k}, \mathrm{p} 1, \mathrm{p} 2, \mathrm{p} 0, \mu$, and $\mathrm{L}$ denote the gas velocity $(\mathrm{m} / \mathrm{s})$, permeability $(\mathrm{m} 2)$, inlet pressure $(\mathrm{Pa})$, outlet pressure $(\mathrm{Pa})$, atmospheric pressure $(101,325 \mathrm{~Pa})$, viscosity of the gas (Pa.s), and flow length (m) of gas, respectively.

As shown in Equation (10), v represents a linear relationship through the origin with $\mathrm{p} 1$ and $\mathrm{p} 2$. With the presence of the threshold pressure gradient, the relationship is expressed as follows:

$$
v=a\left(p_{1}^{2}-p_{2}^{2}\right)-b
$$

Thus, the threshold pressure gradient is

$$
p_{1}=\left(\frac{b}{a}+p_{2}{ }^{2}\right)^{\frac{1}{2}}
$$

The starting pressure gradient is

$$
\lambda=\frac{\left(\frac{b}{a}+p_{2}^{2}\right)^{\frac{1}{2}}-p_{2}}{L}
$$

Therefore, the threshold pressure gradient can be calculated by substituting the constants $a$ and $b$ into Equation (13) after regression on the relationship between $\mathrm{v}$ and $\mathrm{p} 12-\mathrm{p} 22$.

Coal blocks were taken from Daning Mining Area in Qinshui Basin, Shanxi Province, China and processed into eight coal cores measuring $\Phi 50 \mathrm{~mm} \times 50 \mathrm{~mm}$. The test system consists of rubber sealing sleeves, the loading and control system of a testing machine, a high-pressure gas source, and gas flow and pressure testers. The regression method was employed during the test: the gas pressure was adjusted to $18 \mathrm{MPa}$, then the constant pressure valves were closed to test the corresponding flows of a series of pressure gradients. By linear regression between the gas velocity and the difference of squares of pressure, the constants $\mathrm{a}$ and $\mathrm{b}$ in Equation (11) were fitted and then substituted into Equation (13) to calculate the threshold pressure gradient. Furthermore, the permeability was derived according to Equation (10). By fitting the permeability and the threshold pressure gradient, their relationship could be attained. The calculated results of the threshold pressure gradients of different coal samples are listed in Table 1.

Table 1 The calculated results of the threshold pressure gradients of different coal samples

\begin{tabular}{ccccccc}
\hline Test & $\begin{array}{c}\text { Natural dry } \\
\text { weight/g }\end{array}$ & Length/cm & Diameter/cm & Porosity/\% & Permeability/md & $\begin{array}{c}\text { Threshold pressure/10 }^{\mathbf{6}} \\
\text { Pa.m }^{-\mathbf{1}}\end{array}$ \\
\hline A1 & 139.46 & 4.97 & 5.00 & 3.12 & 0.267 & 0.098 \\
\hline
\end{tabular}




\begin{tabular}{lllllcl}
\hline A2 & 137.12 & 4.73 & 5.00 & 3.05 & 2.59 & 0.042 \\
A3 & 140.55 & 5.04 & 5.00 & 2.78 & 0.00897 & 0.614 \\
A4 & 140.81 & 4.98 & 5.00 & 3.14 & 0.013 & 0.497 \\
A5 & 138.64 & 5.00 & 5.00 & 2.91 & 0.815 & 0.0775 \\
A6 & 139.86 & 5.12 & 5.00 & 3.24 & 0.0438 & 0.468 \\
A7 & 140.59 & 4.83 & 5.00 & 2.87 & 0.00241 & 0.76 \\
A8 & 141.7 & 5.01 & 5.00 & 2.97 & 0.0316 & 0.358 \\
\hline
\end{tabular}

The corresponding relationship between the permeability and the threshold pressure gradient is

displayed in Fig. 1.

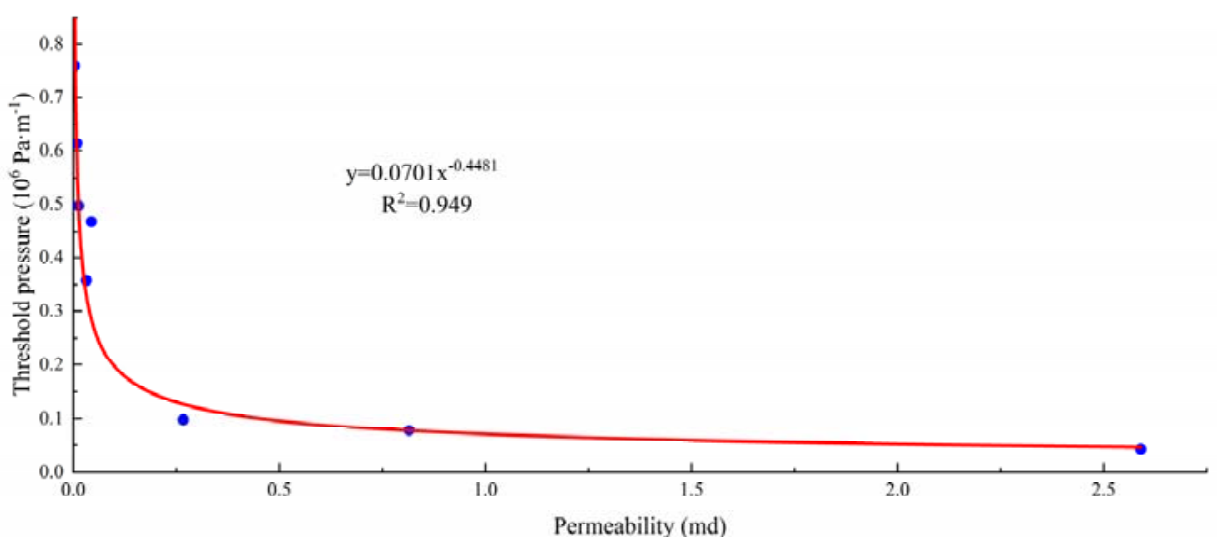

Figure 1 Relation curve between threshold pressure gradient and permeability of coal samples

It can be seen from the figure that the threshold pressure gradient decreases in a negative exponential manner with the increase in permeability.

\subsection{Mathematical model for the reasonable drainage rate in the single-phase flow stage}

By considering the resistance to water flow in coal seams, the flow velocity of water can be expressed as follows (Zhang) ${ }^{[16]}$ :

$$
v=\frac{\bar{k}_{\mathrm{dw}}}{\mu_{\mathrm{w}}}\left(\frac{\Delta p}{\Delta x}-\lambda\right)
$$

where, $\frac{\Delta p}{\Delta x}$ denotes the dynamic gradient $(\mathrm{MPa} / \mathrm{m})$ of fluid flow.

In the single-phase flow stage during drainage, the power required within the range of influence of the water pressure transmission is equal to the product between the dynamic gradient and the radius of influence. In terms of the change of the wellbore pressure, according to the relationship between the permeability and the threshold pressure gradient obtained experimentally, the reduction of the wellbore pressure until time tn can be expressed as follows:

$$
\mathrm{p}_{\mathrm{a}}=0.0701\left(\overline{\mathrm{k}}_{\mathrm{dw}}\right)^{-0.448} \cdot \sqrt{\frac{24 \overline{\mathrm{k}}_{\mathrm{dw}} \mathrm{vt}}{6 \mathrm{v} \emptyset \mu_{\mathrm{w}} \mathrm{C}_{\mathrm{t}}+4 \overline{\mathrm{k}}_{\mathrm{dw}} \mathrm{C \lambda}}}
$$

where, $\mathrm{pa}$ is the reduction of the wellbore pressure until time tn, $\mathrm{MPa} ; \overline{\mathrm{k}}_{\mathrm{dw}}$ is the permeability of reservoirs within the time-step, $\mathrm{mD}$.

In the drainage process, the outflow of water triggers the growth of the effective stress on the coal reservoirs, thus inducing the reduction of the permeability thereof. The dynamic change of the permeability, in turn, can be restricted by the pressure drop of the wellbore and the radius of influence. The dynamic change of the permeability is approximated as a step-wise change by using the method of successive substitution. The average permeability of reservoirs at the start of each time-step is taken as the permeability of reservoirs within the timestep and therefore the dynamic change of the permeability at drainage time tn can be expressed as follows:

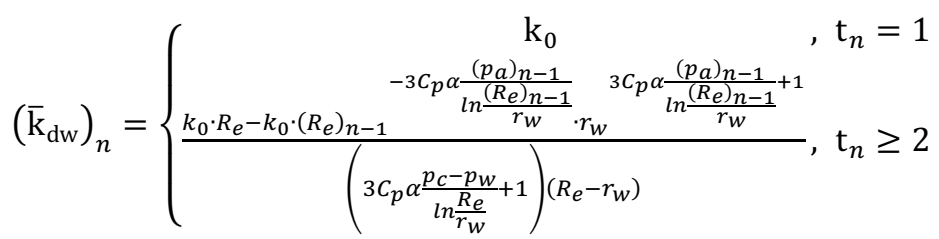

By performing the successive substitution on the

permeability, the resistance gradient, dynamic gradient, 
flow velocity of water in reservoirs, radius of influence of water pressure transmission at drainage time tn, and the pressure drop at the wellbore until time tn are separately calculated as follows:

$$
\begin{gathered}
\lambda_{\mathrm{n}}=0.0701\left(\overline{\mathrm{k}}_{\mathrm{dw}}\right)_{\mathrm{n}}^{-0.448} \\
\mathrm{f}_{\mathrm{n}}=0.034\left(\frac{\left(\overline{\mathrm{k}}_{\mathrm{dw}}\right)_{\mathrm{n}}}{\mu_{w}}\right)^{-0.7115}
\end{gathered}
$$

$$
\begin{aligned}
& v_{n}=\frac{\left(\bar{k}_{d w}\right)_{n}}{\mu_{w}}\left(\mathrm{f}_{\mathrm{n}}-\lambda_{\mathrm{n}}\right) \\
& \operatorname{Re}=\sqrt{\frac{24\left(\bar{k}_{d w}\right)_{n} v_{n} t_{n}}{6 v_{n} \phi \mu C_{t}+4\left(\bar{k}_{d w}\right)_{n} C_{t} \lambda_{n}}} \\
& \left(p_{a}\right)_{n}=0.034\left(\frac{\left(\overline{\mathrm{k}}_{\mathrm{dw}}\right)_{n}}{\mu_{w}}\right)^{-0.7115} \sqrt{\frac{24\left(\overline{\mathrm{k}}_{\mathrm{dw}}\right)_{n} v_{n} t_{n}}{6 \mathrm{v}_{n} \emptyset \mu_{w} \mathrm{C}_{\mathrm{t}}+4\left(\overline{\mathrm{k}}_{\mathrm{dw}}\right)_{n} \mathrm{C}_{t} \lambda_{n}}}(21)
\end{aligned}
$$

The daily reduction value of wellbore pressure is:

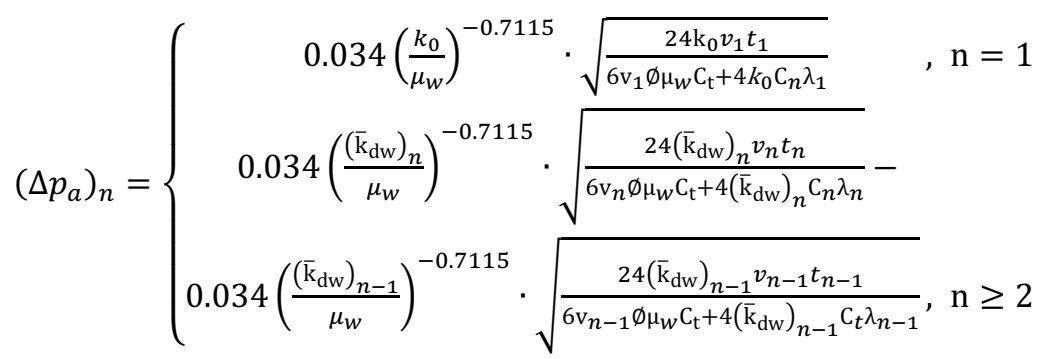

\section{Application and discussion}

\subsection{Application of the reasonable drainage rate obtained based on the established model}

By taking Lanhua Block in Qinshui Basin as an example, the rationality of the drainage system was evaluated. The
No. 3 coal seam in the research area was investigated as it was under development at time of writing. The No. 3 coal seam is an under-pressurised reservoir, with an average thickness of $4.11 \mathrm{~m}$, average gas content of $18.02 \mathrm{~m}^{3} / \mathrm{t}$, average permeability of $1.45 \mathrm{mD}$, average reservoir pressure of $2.14 \mathrm{MPa}$, and reservoir pressure coefficient of between 2.9 to $7.3 \mathrm{kPa} / \mathrm{m}$. Two typical adjacent wells (LD-01 and LD-02) were selected for this analysis: the basic parameters of the typical wells required for the calculation are listed in Table 2.

Table 2 Basic parameters for typical well calculation

\begin{tabular}{ccccccc}
\hline Well & $\mathbf{P}_{\mathbf{0}} / \mathbf{M p a}$ & $\boldsymbol{\varphi} / \boldsymbol{\%}$ & $\mathbf{K}_{\mathbf{0}} / \mathbf{m D}$ & $\mathbf{C}_{\mathbf{t}} / \mathbf{M p a}^{-\mathbf{1}}$ & $\mathbf{C}_{\mathbf{p}} / \mathbf{M p a}^{-\mathbf{1}}$ & $\boldsymbol{\mu} / \mathbf{m P a} \cdot \mathbf{s}$ \\
\hline LD-01 & 3.185 & 1.5 & 0.068 & 0.004 & 0.0005 & 1.005 \\
LD-02 & 2.982 & 5 & 2.3 & 0.004 & 0.0005 & 1.005 \\
\hline
\end{tabular}

According to the basic parameters in Table 2, the reasonable rates of the pressure drop of the two wells in the single-phase flow stage are calculated and compared with actual drainage curves.

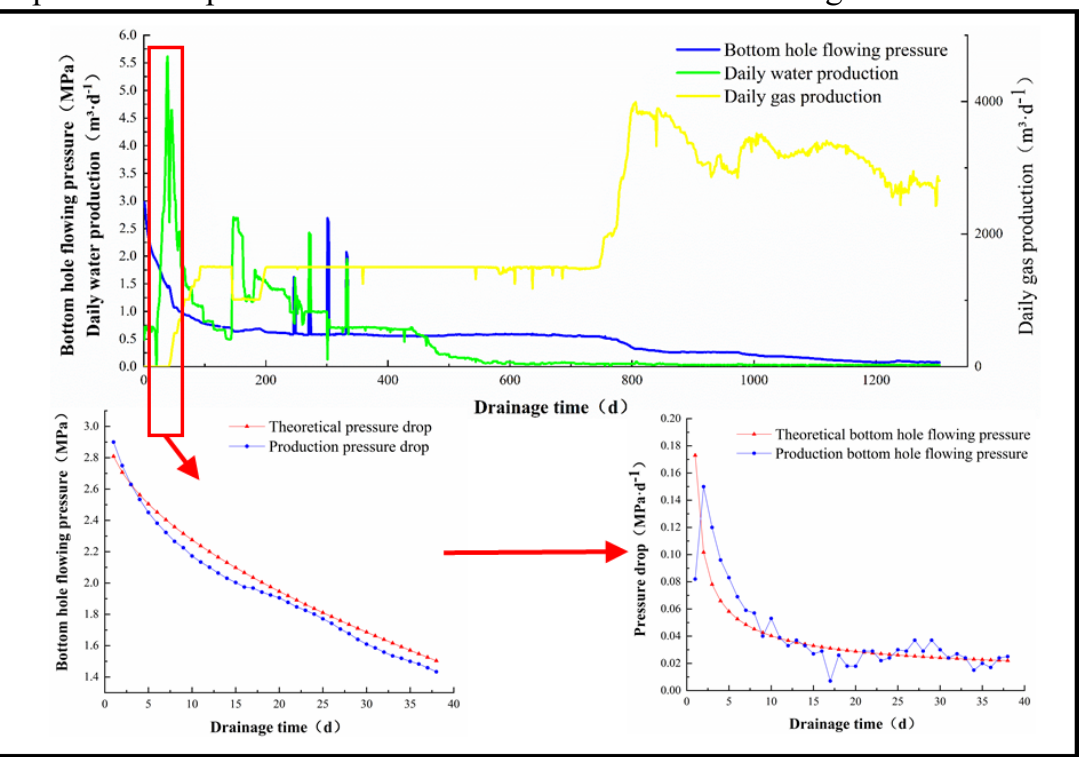

Figure 2 Pressure drop comparison of LD-01 well in single phase flow stage 


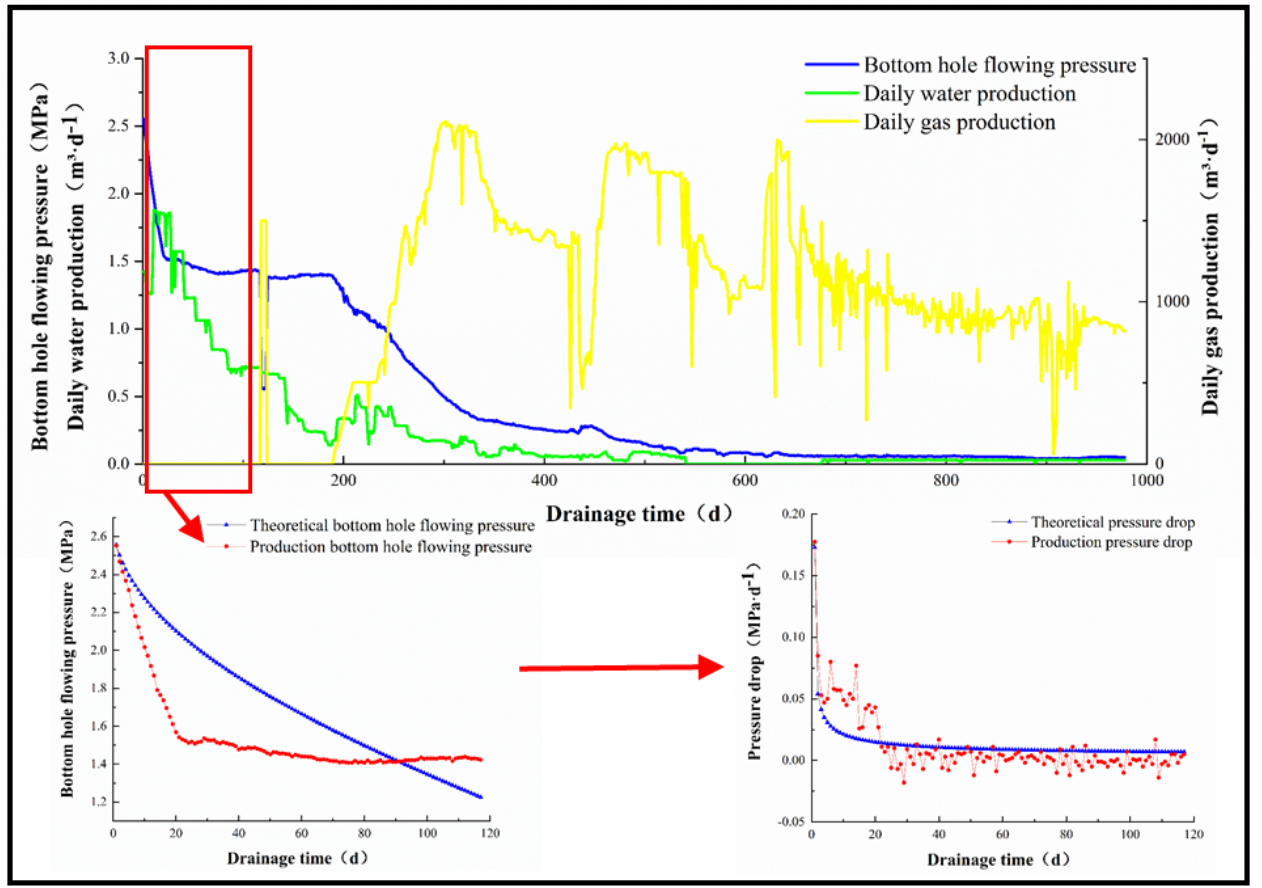

Figure 3 Pressure drop comparison of LD-02 well in single phase flow stage

As shown in Figs 2 and 3, the drainage system of the well LD-01 in the single-phase flow stage conforms to the reasonable drainage rate calculated by using the proposed method. The daily gas production in the stable production stage can reach about $4000 \mathrm{~m}^{3} / \mathrm{d}$, showing favourable gas production conditions; the drainage system of the well LD-02 in the single-phase flow stage greatly differs from the reasonable drainage rate calculated by applying the proposed method. The difference mainly appears as an excessive rate of pressure drop and significant fluctuations in water production in the initial drainage stage, which causes the pulverised coal to be transported and output to block fractures in reservoirs, thus lowering the permeability of the coal seams. The daily gas production in the stable production stage is unstable, fluctuating within the range of 1000 to $2000 \mathrm{~m}^{3} / \mathrm{d}$, delivering poor gas production conditions. This validates the accuracy of the theoretical model to some extent.

\subsection{The reasonable rates of pressure drop of CBM wells under different permeabilities}

According to the established mathematical model for the pressure drop, the reasonable rates of pressure drop under permeabilities of $0.2,0.4,0.6,0.8,1.0,1.5$, and $2.0 \mathrm{mD}$ are separately calculated (Fig. 4)

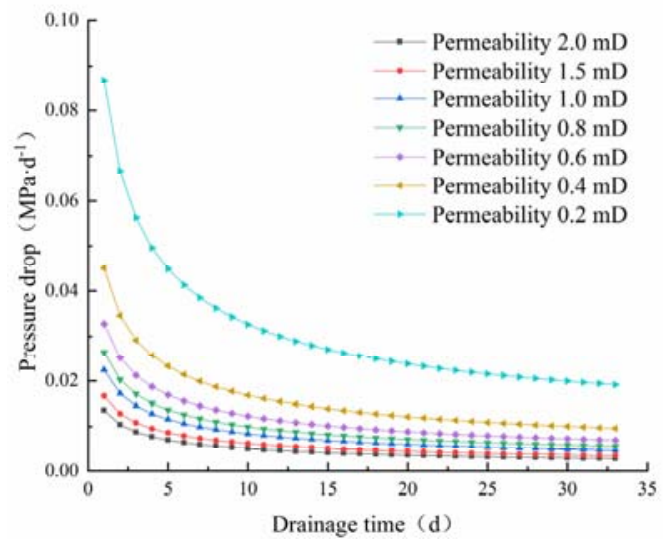

Figure 4 Calculation of reasonable pressure drop rate under different permeability

As shown in Fig. 4, the reasonable rate of pressure drop reduces in a negative logarithmic manner with drainage time, that is, a large pressure drop is required in the initial CBM drainage stage. As drainage continues, the 
increase in transmission distance of the water pressure slows down and the pressure required for increasing the distance decreases; moreover, the pressure drop also decreases. The reasonable rates of pressure drop differ under different permeabilities. The larger the permeability, the lower the threshold pressure gradient and the lower the pressure drop required for daily drainage.

\section{Conclusion}

(1) According to the linkage mechanism of bottom-hole pressure change, pressure propagation distance and permeability change in the drainage and production process, a mathematical model for the reasonable rate of fluid level decline in the single phase flow with permeability change was established. The accuracy of the mathematical model is verified by taking the actual drainage data of orchid block in Qinshui Basin as an example.

(2) Based on the basic parameters of the reservoir in The Orchid block of Qinshui Basin, the reasonable pressure drop rate of coalbed methane Wells under different permeability conditions is calculated. The results show that the pressure drop rate decreases in negative exponential form with the prolongation of drainage time in single-phase flow stage. Under the same other conditions, the greater the permeability of coal reservoir, the smaller the start-up pressure gradient and the smaller the daily pressure drop required for coal drainage and mining.

\section{Acknowledgements}

This study was supported by the Program for Innovative Research Team (in Science and Technology) in Universities of Henan Province, China (Grant No.21IRTSTHN007) and The Program for Innovative Research Team (in Science and Technology) of Henan Polytechnic University (GrantNo.T2020-4).

\section{References}

1. Ni Xiaoming, Wang yanbin, Jie Mingxun, Wu Jianguang, Reasonable production intensity of coalbed methane wells in initial production. Journal of Southwest Petroleum University(Science \& Technology Edition), 06 (2007).

2. Yi Yongxiang, Tang Shuheng, Zhang Songhang, Yan Xinlu, Wang Kaifeng, Dang Feng. Analysis on the type of reservoir pressure drop and drainage control of coalbed methane well in the southern block of Shizhuang, Coal Geology \& Exploration, 05 (2019).

3. Ni Xiaoming, Zhang Chongchong, Wang Yanbin, Wang Xianghao. Mathematical models about the critical size of coal reservoir fracture make of water migration for single-phase coalbed methane wells. Engineering Mechanics, 04 (2015).

4. Kang Yongshang, Qin Shaofeng, Han Jun, Diao Shun, Mao Delei, Dou Fengke. Typical dynamic index analysis method system for drainage and production dynamic curves of CBM wells, Journal of China Coal Society, 10 (2013).

5. Deng Ze, Kang Yongshang, Liu Honglin, Li Guizhong, Wang Bo. Dynamic variation character of coal bed methane reservoir permeability during depletion. Journal of China Coal Society, 07(2009).

6. Meng Yanjun, Tang Dazhen, Li Zhiping, Xu Hao, Tao Shu, Li Song. Dynamic variation characteristics and mechanism of permeability in high-rank CBM wells at different drainage and production stages. Petroleum Geology and Recovery Efficiency, 02 (2015).

7. LI Guoqing, Meng Zhaoping, Wang Baoyu. Diffusion and seepage mechanisms of high rank coal-bed methane reservoir and its numerical simulation at early drainage rate. Journal of China Coal Society, 09 (2014).

8. Guo Chunhua, Zhou Wen, Sun Hansen, Yang Yong. The relationship between stress sensitivity and production of coal bed methane wells. Coal Geology \& Exploration, 05 (2011).

9. Wu Caifang, Yao Shuai, Du Yanfei. Production systems optimization of a CBM well based on a time series BP neural network. Journal of China University of Mining \& Technology, 01 (2015).

10. Yao Yuedong, Ge Jiali, Li Xiangfang. Oil-water twophase fluid flow in low permeability reservoir. Journal of China University of Petroleum(Edition of Natural Science), 02 (2005).

11. Yao Yuedong, Ge Jiali, Wei Junzhi. Study on the fluid flow in low permeability reservoir. Petroleum Exploration and Development, 04 (2001).

12. Zhu Shengju. The Propagation of Pressure Wave from Low Permeability Reservoirs. Xinjiang Petroleum Geology, 01 (2007).

13. Zhu Shengju. The Law of Pressure Spread in Different Flow States. Xinjiang Petroleum Geology, 06 (2002).

14. Chen Shida, Tang Dazhen, Gao Lijun, Xu Hao, Zhao Junlong, Tao Shu. Control of effective stress on permeability in high-rank coal reservoirs. Coal Geology \& Exploration, 04 (2017).

15. Zou Xing, Wu fan, Sun Lijuan, Qiao Guo. Method of establishing two-phase starting pressure curve. Petroleum Exploration and Development, 03 (2004).

16. Zhang Lei.The Study of Transmission of Pressure in Low-permeable Reservoir.China University of petroleum,2009. 